Article

\title{
Microwave-Absorbing Properties of Rice Starch
}

\author{
Daming Fan ${ }^{1}$, Huijie Shen ${ }^{1}$, Luelue Huang ${ }^{2}$, Yishu Gao ${ }^{1}$, Huizhang Lian ${ }^{3}$, Jianxin Zhao ${ }^{1}$, \\ Hao Zhang ${ }^{1}$ and Wei Chen ${ }^{1,4, *}$
}

${ }^{1}$ State Key Laboratory of Food Science and Technology, School of Food Science and Technology, Jiangnan University, Wuxi 214122, China; E-Mails: fandm@jiangnan.edu.cn (D.F.); shenhuijiewu@163.com (H.S.); 7140112010@vip.jiangnan.edu.cn (Y.G.); jxzhao@jiangnan.edu.cn (J.Z.); zhanghao@jiangnan.edu.cn (H.Z.)

${ }^{2}$ School of Applied Chemistry and Biological Technology, Shenzhen Polytechnic, Shenzhen 518055, China; E-Mail: huangluelue@126.com

${ }^{3}$ Wuxi Huashun Minsheng Food Co. Ltd., Wuxi 214218, China;

E-Mail: lhz13616170044@ hotmail.com

${ }^{4}$ Beijing Innovation Centre of Food Nutrition and Human Health, Beijing Technology \& Business University, Beijing 100048, China

* Author to whom correspondence should be addressed; E-Mail: chenwei66@jiangnan.edu.cn; Tel.: +86-0510-8588-4620; Fax: +86-0510-8591-2155.

Academic Editor: Katja Loos

Received: 20 July 2015 / Accepted: 17 September 2015 / Published: 22 September 2015

\begin{abstract}
In the food industry, research into the characteristics of microwave-heated materials has focused on dielectric properties. However, the lack of studies on microwave-absorbing properties has hindered the application of microwave technology. The aim of the present study was to investigate the microwave-absorbing properties of rice starch. It should be noted that this was the first time that the improved arch method was used to measure the microwave reflection loss (RL) of the starch dispersion. The results showed that the microwave absorption of the liquid system corresponded to the classical quarter-wavelength resonator model. When the concentration of the native starch was increased from $1 \%$ to $30 \%$, the RL decreased from $-5.1 \mathrm{~dB}$ to $-1.2 \mathrm{~dB}$ at $2.45 \mathrm{GHz}$. Therefore, the absorption rate of microwave, $\gamma_{\mathrm{ab}}$, decreased from $69.1 \%$ to $24.1 \%$. At 1.7 to $2.6 \mathrm{GHz}$, the interference effect of pregelatinization rice starch in an aqueous system on the microwave absorption properties became weak compared to native starch.
\end{abstract}


Keywords: rice starch; microwave-absorbing properties; improved arch method

\section{Introduction}

The response of a material in a microwave field cannot be described by its complex permittivity alone, as it can also be affected by the size, resistivity and complex permeability, among others. The response of starch to electromagnetic waves determines the absorption, release and loss ability of the microwave. The absorbing properties of the material were the comprehensive performance of its dielectric properties, the magnetic properties and its macroscopic morphology. Therefore, the study of the microwave-absorbing properties of starch can be used to describe the response of the material to microwaves more completely and also to predict the effect on the space field density. Therefore, the study of the microwave absorption properties of starch materials at low power electromagnetic fields based on a vector network analyzer can provide the theoretical basis to investigate the effect of high power microwave on starch material and to realize the application of high power microwave in the processing field.

The reflection loss (RL) of an electromagnetic wave can be measured using the arch method [1-3], which has been widely used in the study of absorbing materials in military areas. The absorption rate can be further calculated using the RL value.

Potato starch dielectric properties have been shown to change significantly with changes in temperature and moisture [4]. The dielectric loss factor, $\varepsilon "$, of gelatinized potato starch is higher than for granules [5]. The permittivity of starch was measured over a 0.2 to $20 \mathrm{GHz}$ frequency range [6]. The structure of gelatinized and swollen starch combined with little water. More water was therefore in a free state and responded to the changes in the microwave field, generating heat. With an increase in temperature, the change in permittivity $\varepsilon$ ' and $\varepsilon$ " of potato, wheat, corn and waxy cornstarch suspensions decreased, and with a decrease in concentration, $\varepsilon^{\prime}$ increased, whereas $\varepsilon$ " changed only little [7]. A study of the effect of temperature on the dielectric properties of five types of starch, including both granular and suspension forms, showed that with increasing temperature, $\varepsilon^{\prime}$ and $\varepsilon$ " of the granular starch increased, whereas for starch in solution, $\varepsilon^{\prime}$ and $\varepsilon$ " decreased [4]. Research on the electromagnetic properties of starchy foods has primarily focused on dough $[8,9]$.

However, at present, research on the microwave absorption of food ingredients is scarce, significantly limiting the application of industrial and civilian microwave technology in this field. In this study, to determine the production and reheating adjustments of microwaved rice products, the microwave-absorbing properties of both rice starch granules and its gelatinized form in an aqueous system were investigated. The electromagnetic properties of starch in a microwave field were tested to determine the loss type. We explored an innovative approach to measuring the starch RL, for the further study of its microwave-absorbing properties. 


\section{Materials and Methods}

\subsection{Materials}

2.1.1. Preparation of Starch and Rice Starch Dispersion

Rice starch (protein content $\leqslant 0.23 \%$, starch content $\geqslant 94.22 \%$, amylose content $15.11 \%$, particle size $5.52 \pm 0.23 \mu \mathrm{m}$ ) was isolated and purified from fresh rice (QiuShouBao Rice Products Co., Ltd., Huoqiu, Anhui, China), as described previously [10,11]. Dispersions of rice starch at 1\%, 5\%, 7\%, $8 \%$ or $9 \%$ $(w / w)$ concentrations were prepared by adding deionized water at room temperature.

\subsubsection{Preparation of Samples in an Amorphous State}

Amorphous starch was prepared as described previously [12].

\subsection{Methods}

\subsubsection{Electromagnetic Measurements}

The electromagnetic parameters of starch granules were measured using the coaxial transmission line testing method [13]. Coaxial specimens for the electromagnetic parameters were prepared by dispersing starch samples in molten paraffin wax, then molding it into toroid-shaped samples with a 7.00-mm outer diameter and a 3.04- $\mathrm{mm}$ inner diameter. The specimens were surveyed over a sweeping range of 2 to $7 \mathrm{GHz}$. The vector network analyzer (HP 8720B, Hewlett Packard Co., Palo Alto, CA, USA) was the most important part of the testing system. The vector network analyzer was calibrated before measuring, and the sample was then set in the test fixture. The electromagnetic parameters, via the transmission method, were calculated from the scattering parameters.

\subsubsection{Dielectric Measurements}

The dielectric properties of the liquid system were measured using a vector network analyzer (E5071C, Agilent, Santa Clara, CA, USA) with an open-ended coaxial line, connected to a dielectric probe (85070E, Agilent, Santa Clara, CA, USA) [14,15]. The probe was first calibrated using three materials, air, water (of known temperature) and metal, to ensure the accuracy of the measurements. Each sample was mixed to homogeneity before testing and was measured in triplicate.

\subsubsection{RL Measurements}

The RL versus frequency for complex liquid materials was improved on the basis of the arch method by adding polytetrafluoroethylene (PTFE) as the wall material to the former aluminum plate, to make a specialized container (Figure 1a). The testing system of the improved arch method included a vector network analyzer (HP 8720B, Hewlett Packard Co., Palo Alto, CA, USA) and standard horn antennas in an anechoic chamber as a function of frequency from 1.7 to $2.6 \mathrm{GHz}$ (Figure $1 \mathrm{~b}$ ). 
(a)

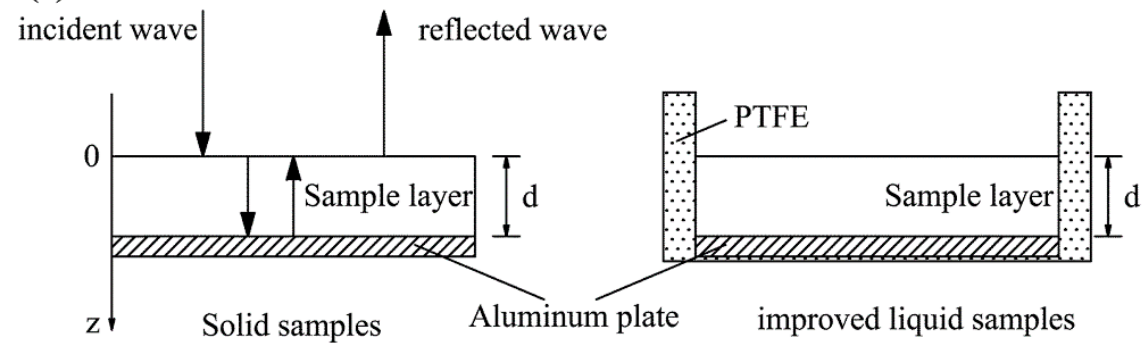

(b)

\section{Network analyzer \\ 2. Transmitter \\ 3. Receiver \\ 4. Reflecting plate \\ 5. Pyramidal absorber \\ 6. Transmission line \\ 7. Arch beam}

\section{6}

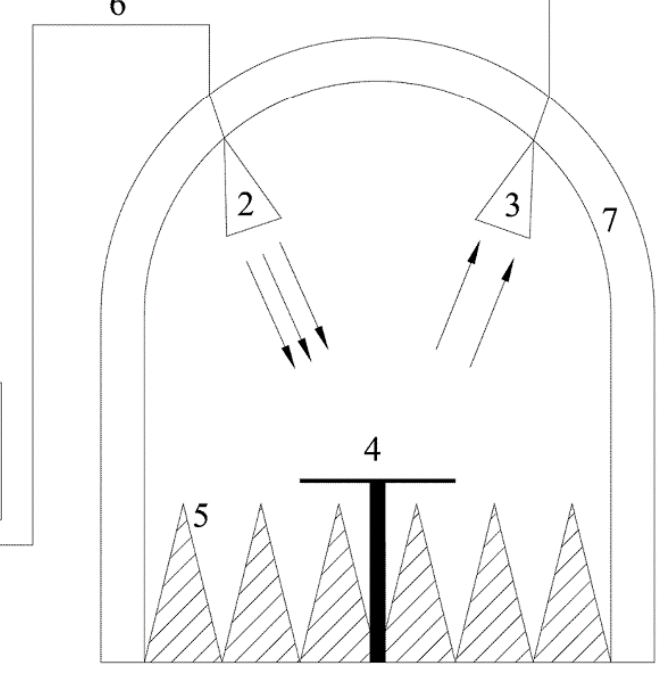

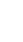

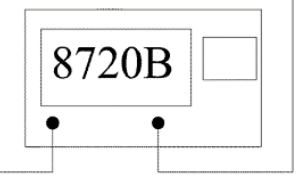

Figure 1. (a) The improved specialized container for liquid samples and (b) the schematic diagram of the arch method testing system.

\section{Results and Discussion}

\subsection{The Electromagnetic Properties of Rice Starch}

As shown in Figure 2a,b, at a range of 2 to $7 \mathrm{GHz}, \varepsilon$ and the permeability, $\mu$, of the two samples fluctuated periodically and were nearly constant with the increase in frequency. For the complex permittivity of both native and completely gelatinized starch, $\varepsilon^{\prime}$ was $2.7 \pm 0.1$, and $\varepsilon$ " lay in the range of 0 to 0.2 , with $\tan \delta_{\varepsilon}$ below 0.1 , indicating that rice starch weakly absorbs microwaves. For complex permeability, $\mu$ ' was $1.00 \pm 0.10$, and $\mu$ " and $\tan \delta_{\mu}$ were both $0.19 \pm 0.02$, indicating weak magnetic induction. Compared to native starch, the complex permittivity of gelatinized starch in Figure $2 \mathrm{c}$ did not change significantly, indicating that the solid powder of each was not obviously polarized. From the testing data, we conclude that starch experiences dielectric loss.

\subsection{The Dielectric Properties of the Rice Starch Dispersion}

We prepared a range of concentrations of the starch dispersion, $1 \%, 5 \%, 7 \%, 8 \%$ and 9\% [16,17], to study $\varepsilon^{\prime}$ and $\varepsilon^{\prime \prime}$ at room temperature and at $2.45 \mathrm{GHz}$. The results are shown in Figure 3 . With the increase in concentration, the $\varepsilon$ ' of the starch dispersion decreased in a linear manner, whereas $\varepsilon$ " showed little change, indicating that the ability of the starch to absorb and store the microwaves in the microwave field declined, but its ability to transform the microwave energy into heat did not, which is consistent with previous reports [7]. 

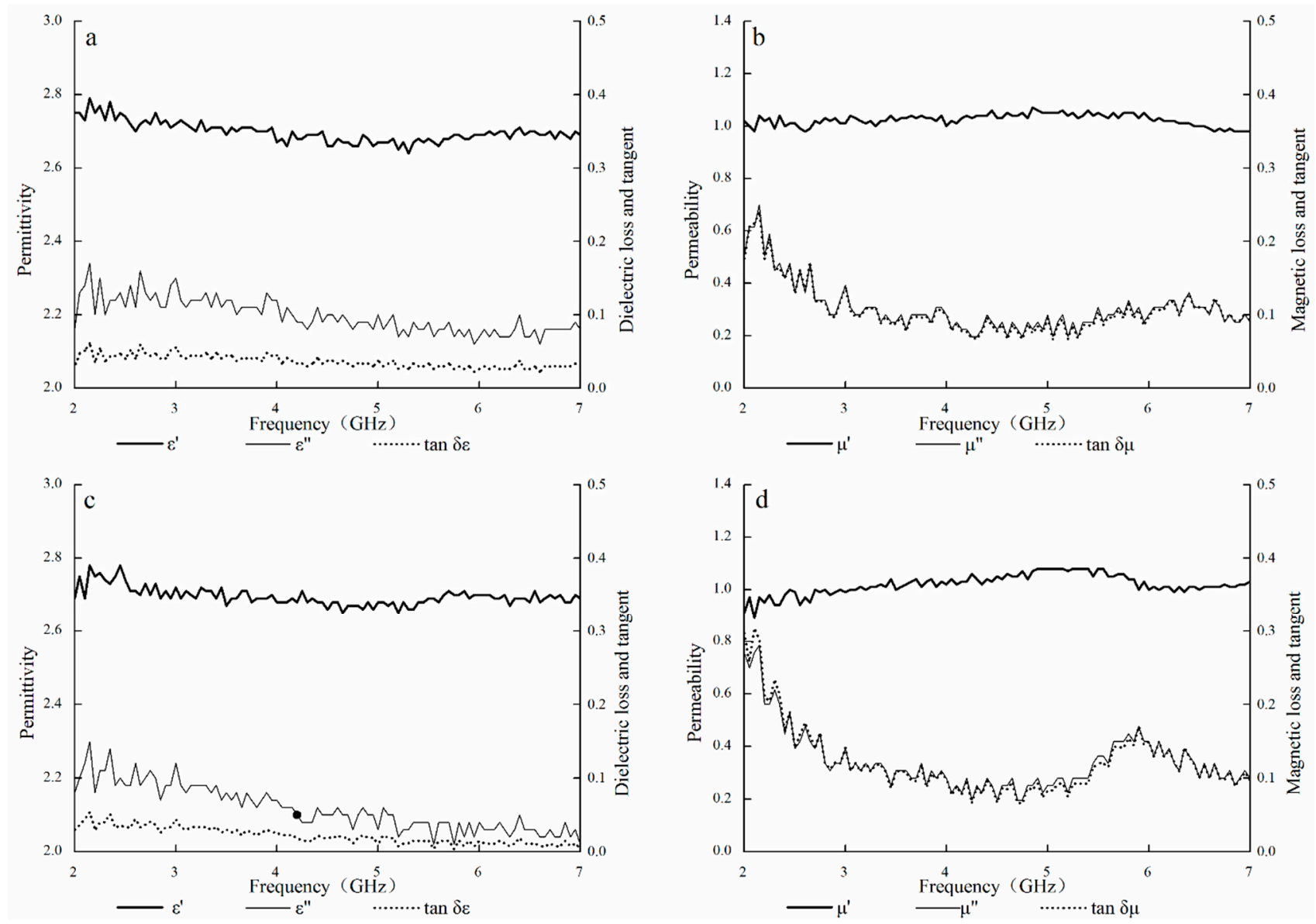

Figure 2. The complex dielectric permittivity values (a,c) and complex permeability values (b,d) of rice starch and gelatinized starch, respectively, in the range of 2 to $7 \mathrm{GHz}$, at room temperature.

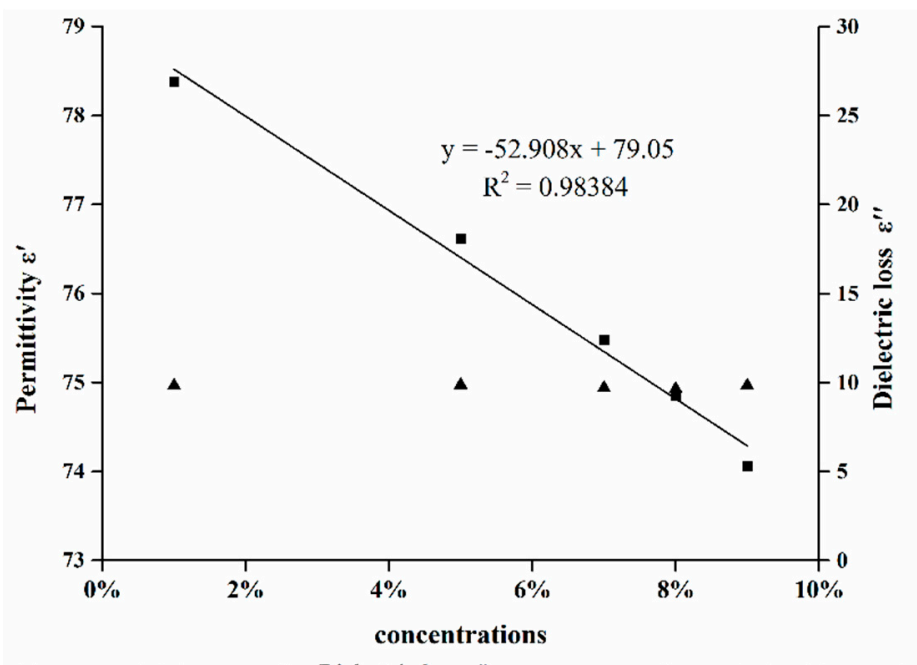

- Permillivily $\varepsilon^{\prime}$ linear relationship(permittivity)

Figure 3. The permittivity ( $\mathbf{\square}$ ) and dielectric loss ( $\mathbf{\Delta})$ values for starch dispersions of different concentrations at $2.45 \mathrm{GHz}$, at room temperature. 


\subsection{RL of Water}

To determine whether the improved arch method can be applied to the liquid material, we examined the RL of the water system at different thicknesses and at a frequency of $2.45 \mathrm{GHz}$. We calculated $1 / 4$ of the electromagnetic wavelength, $\lambda_{\mathrm{m}}$, of the water system to be $3.23 \mathrm{~mm}$, according to the following formula:

$$
\lambda_{\mathrm{m}}=\frac{\lambda_{0}}{\sqrt{\mu \varepsilon}}
$$

where $\varepsilon$ and $\mu$ of the water system, as well as the wavelength in a vacuum spread, $\lambda_{0}$, are $79.05 \pm 0.01$, $1.06 \pm 0.01$ and about $0.12245 \mathrm{~m}$, respectively at $2.45 \mathrm{GHz}$ and $24 \pm 1{ }^{\circ} \mathrm{C}$. As shown in Figure 4, the maximum value of RL, or the largest microwave absorption of the water system, occurred at a $3 \mathrm{~mm}$ water thickness, which corresponded to the classical quarter-wavelength $(\lambda / 4)$ resonator model [13]. The formula of the resonator model can be expressed as follows:

$$
f_{\mathrm{m}}=\frac{n c}{4 d \sqrt{\varepsilon \mu}}(n=1,3,5, \ldots)
$$

where $d, \varepsilon$ and $\mu$ denote the thickness, relative complex permittivity and permeability of the testing materials, respectively; $\mathrm{c}$ is the velocity of light, and the peak frequency of the test material is defined as matching the frequency $f_{\mathrm{m}}$. According to the resonator model, when the thickness of the material is equal to the value of $1 / 4$ of the electromagnetic wavelength, interference effects between the wave and the material occur, leading to an energy decline in the reflected electromagnetic wave. We conclude that the improved arch method is suitable for the determination of the microwave-absorbing properties of a liquid system.

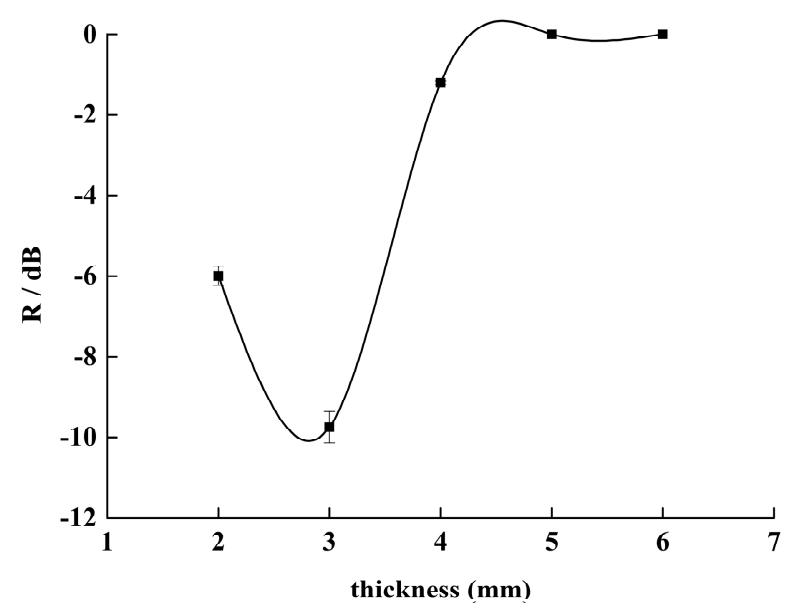

Figure 4. The RL curve of a water system of varying thickness at $2.45 \mathrm{GHz}$, at room temperature.

\subsection{Microwave-Absorbing Properties of the Rice Starch Dispersion}

We investigated the RL curves of $3 \mathrm{~mm}$-thick starch-water mixtures at different concentrations. As shown in Figure 5a, the starch dispersion exhibited regular changes, and significantly, the location of the absorption peak was at the same location at frequencies of 1.7 to $2.6 \mathrm{GHz}$. In addition, there was little change in the interval of the RL peak compared to Figure $5 \mathrm{~b}$. These results suggest that water molecules dominated the electromagnetic properties of the rice starch dispersion, whereas the starch had 
little effect on the value of the RL peak. Microwave heating is greatly affected by the presence of water in foods $[18,19]$. The 3-mm thickness of the rice starch dispersion was appropriate to reflect the change in the absorption of the whole system.
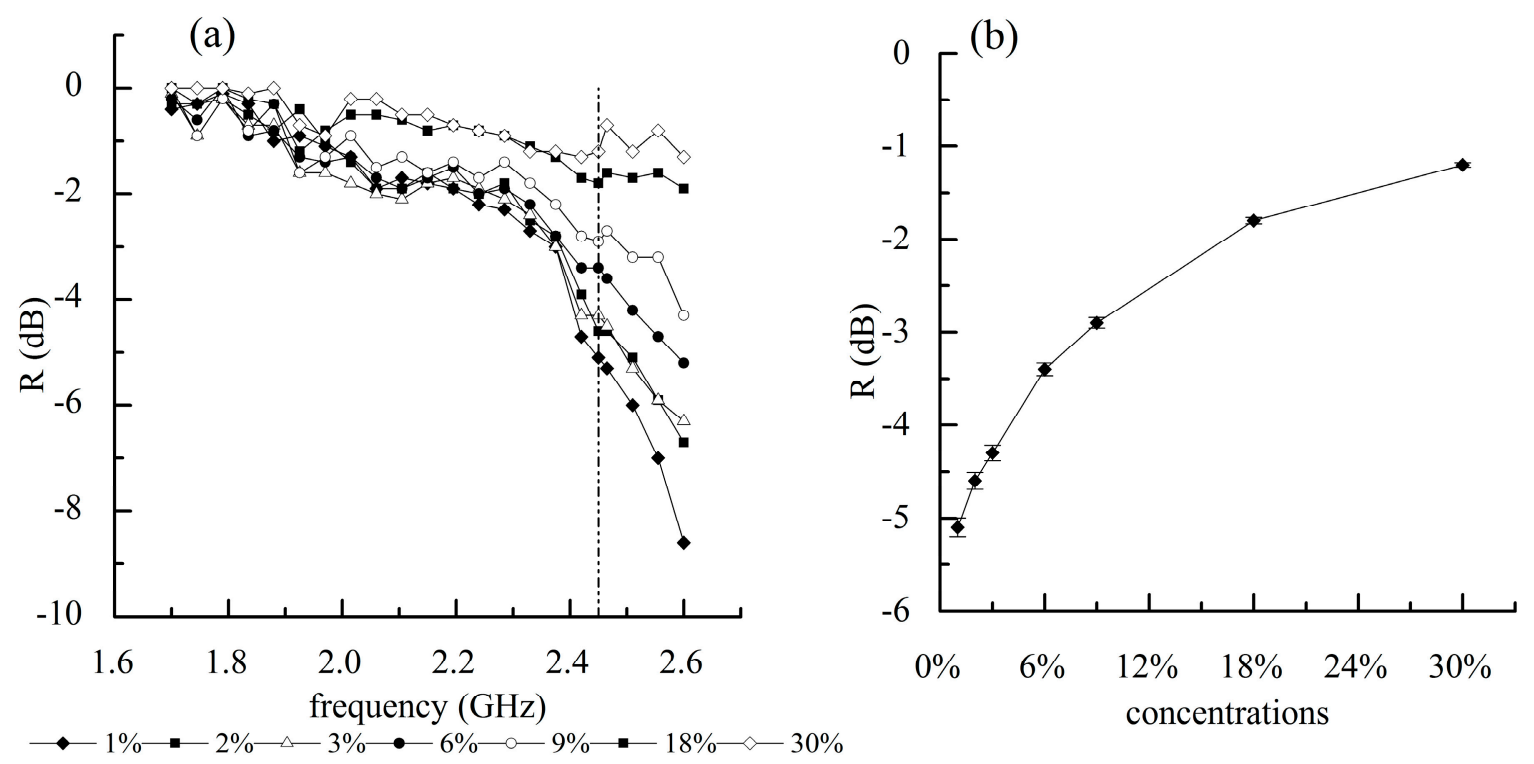

Figure 5. (a) The RL patterns in the frequency range 1.7 to $2.6 \mathrm{GHz}$ and (b) the RL curve at $2.45 \mathrm{GHz}$ for starch dispersions of different concentrations at room temperature.

From Figure $5 b$, it can be seen that when the concentration of the native starch increased from $1 \%$ to $30 \%$, the RL decreased from $-5.1 \mathrm{~dB}$ to $-1.2 \mathrm{~dB}$ at a frequency of $2.45 \mathrm{GHz}$. Therefore, the absorption rate of the electromagnetic wave, $\gamma_{\mathrm{ab}}$, decreased from $69.1 \%$ to $24.1 \%$, calculated using the following formula:

$$
\gamma_{\mathrm{ab}}=-\frac{1}{\sqrt[10]{10^{|R L|}}}
$$

Considering the homogeneity of the samples during processing, we discuss the absorption of $6 \%$ native and pregelatinization rice starch. As shown in Figure 6, although accounting for only a small proportion, the native starch significantly interfered with the microwave absorption properties of the water, whereas the properties of the whole mixture were less affected by the pregelatinization rice starch. At a frequency of 1.7 to $2.6 \mathrm{GHz}$, the type and height of the absorption peak for the pregelatinization rice starch system was consistent with the water system, indicating that water played the leading role in the microwave absorption. The detailed data are shown in Table 1.

Table 1. Detailed data on the absorption performance of starch emulsions.

\begin{tabular}{cccc}
\hline Samples & Water & 6\% Native starch & 6\% Pregelatinization starch \\
\hline Peak value $(\mathrm{dB})$ & -12.95 & -6.3 & -10.5 \\
Absorption frequency band $(\mathrm{GHz}) \mathrm{RL}<-8 \mathrm{~dB}$ & $2.42 \sim 2.60$ & - & $2.44 \sim 2.60$ \\
Absorption frequency band $(\mathrm{GHz}) \mathrm{RL}<-8 \mathrm{~dB}$ & $2.42 \sim 2.60$ & - & 0.16 \\
\hline
\end{tabular}




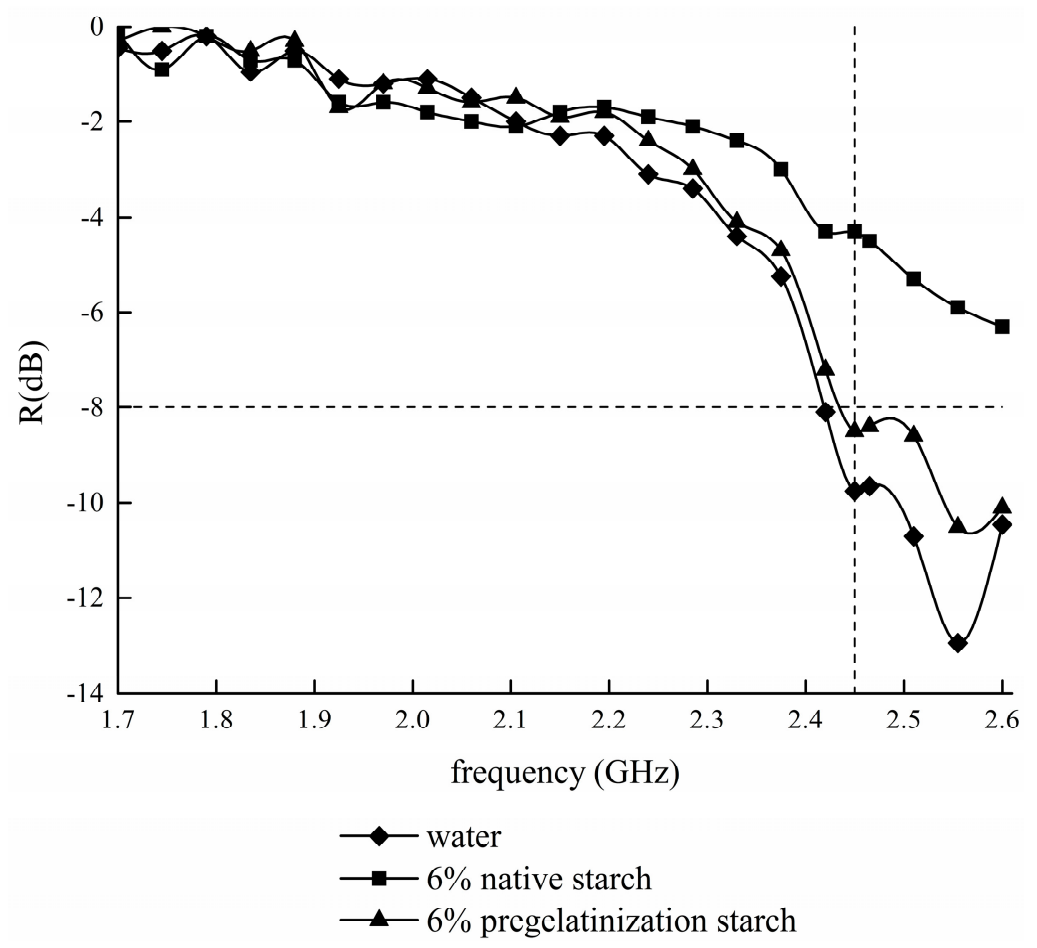

Figure 6. The RL curves of native and pregelatinization starch-water mixtures of a $6 \%$ concentration.

\section{Conclusions}

The microwave-absorbing properties of rice starch were investigated indirectly from the point of view of both the electromagnetic characteristics and the size effect. It can be concluded that rice starch is a weakly-absorbing material of weak electromagnetic induction. It showed the greatest microwave absorption for $3 \mathrm{~mm}$-thick water, indicating that the interference effects between microwave and water were consistent with the classical quarter-wavelength $(\lambda / 4)$ resonator model.

The microwave-absorbing properties of rice starch were investigated directly for the first time to use the improved arch method to measure the RL of the starch dispersion. When the concentration of the starch dispersion was increased from $1 \%$ to $30 \%$, the microwave-absorbing rate decreased from $69.1 \%$ to $24.1 \%$. At a frequency of 1.7 to $2.6 \mathrm{GHz}$, the interference effect of pregelatinization rice starch in an aqueous system on the microwave absorption properties became smaller compared to the native starch. Further studies will be required to determine the different effects of microwave and traditional heating methods on the microwave absorption of rice starch and to take advantage of the relevant models to explain the material interference of the microwave space field.

\section{Acknowledgement}

This research was supported by National Natural Science Foundation of China (Grant No. 31301504, No. 31571879), the Natural Science Foundation of Jiangsu Province (Grant No. BK20130141) and the Doctoral Program of the Higher Education Research Fund (For New Teachers, Grant No. 20130093120011). 


\section{Author Contributions}

Daming Fan, Huijie Shen and Luelue Huang designed experiments; Yishu Gao and Daming Fan carried out experiments; Hao Zhang, Jianxin Zhao, Huizhang Lian and Wei Chen analyzed the data and developed analysis tools; Daming Fan and Huijie Shen wrote the manuscript.

\section{Conflicts of Interest}

The authors declare no conflict of interest.

\section{Abbreviations}

RL, reflection loss $(\mathrm{dB})$; $\varepsilon$, relative complex permittivity; $\varepsilon^{\prime}$, permittivity; $\varepsilon^{\prime \prime}$, dielectric loss factor; $\tan \delta_{\varepsilon}$, dielectric loss tangent; $\mu$, relative complex permeability; $\mu$ ', permeability; $\mu$ ", magnetic loss factor; $\tan \delta_{\mu}$, magnetic loss tangent; $\lambda_{\mathrm{m}}$, electromagnetic wavelength $(\mathrm{m}) ; \lambda_{0}$, wavelength in a vacuum spread $(\mathrm{m}) ; \mathrm{d}$, thickness $(\mathrm{mm}) ; f_{\mathrm{m}}$, matching frequency $(\mathrm{GHz}) ; \gamma_{\mathrm{ab}}$, absorption rate of the electromagnetic wave; $c$, the velocity of light (m).

\section{References}

1. Montgomery, C.G. Technique of Microwave Measurements; McGraw-Hill Book Co.: New York, NY, USA, 1947.

2. Feng, Y.B.; Qiu, T.; Shen, C.Y. Absorbing properties and structural design of microwave absorbers based on carbonyl iron and barium ferrite. J. Magn. Magn. Mater. 2007, 318, 8-13.

3. Ting, T.H.; Yu, R.P.; Jau, Y.N. Synthesis and microwave absorption characteristics of polyaniline/NiZn ferrite composites in 2-40 GHz. Mater. Chem. Phys. 2011, 126, 364-368.

4. Nelson, S.; Prakash, A.; Lawrence, K. Moisture and temperature-dependence of the permittivities of some hydrocolloids at $2.45 \mathrm{GHz}$. J. Microw. Power Electromagn. Energy 1991, 26, 178-185.

5. Roebuck, B.D.; Goldblith, S.A.; Westphal, W.B. Dielectric properties of carbohydrate-water mixtures at microwave frequencies. J. Food Sci. 1972, 37, 199-204.

6. Haynes, L.C.; Locke, J.P. Microwave permittivities of cracker dough, starch and gluten. J. Microw. Power Electromagn. Energy 1995, 30, 124-131.

7. Ryynanen, S.; Risman, P.O.; Ohlsson, T. The dielectric properties of native starch solutions-A research note. J. Microw. Power Electromagn. Energy 1996, 31, 50-53.

8. Zuercher, J.; Hoppie, L.; Lade, R.; Srinivasan, S.; Misra, D. Measurement of the complex permittivity of bread dough by an open-ended coaxial line method at ultrahigh frequencies. J. Microw. Power Electromagn. Energy 1990, 25, 161-167.

9. Kim, Y.R.; Cornillon, P. Effects of temperature and mixing time on molecular mobility in wheat dough. LWT Food Sci. Technol. 2001, 34, 417-423. [CrossRef]

10. Fan, D.M.; Ma, W.R.; Wang, L.Y.; Huang, J.L.; Zhao, J.X.; Zhang, H.; Chen, W. Determination of structural changes in microwaved rice starch using Fourier transform infrared and raman spectroscopy. Starch Stärke 2012, 64, 598-606. [CrossRef]

11. Fan, D.M.; Ma, S.Y.; Wang, L.Y.; Zhao, J.X.; Zhang, H.; Chen, W. Effect of microwave heating on optical and thermal properties of rice starch. Starch Stärke 2012, 64, 740-744. [CrossRef] 
12. Fan, D.M.; Ma, W.R.; Wang, L.Y.; Huang, J.L.; Zhang, F.M.; Zhao, J.X.; Zhang, H.; Chen, W. Determining the effects of microwave heating on the ordered structures of rice starch by NMR. Carbohydr. Polym. 2013, 92, 1395-1401. [CrossRef] [PubMed]

13. Duan, Y.P.; Wu, G.L.; Gu, S.C.; Li, S.Q.; Ma, G.J. Study on microwave absorbing properties of carbonyl-iron composite coating based on PVC and Al sheet. Appl. Surf. Sci. 2012, 258, 5746-5752.

14. Miura, N.; Yagihara, S.; Mashimo, S. Microwave dielectric properties of solid and liquid foods investigated by time-domain reflectometry. J. Food Sci. 2003, 68, 1396-1403. [CrossRef]

15. Motwani, T.; Seetharaman, K.; Anantheswaran, R.C. Dielectric properties of starch slurries as influenced by starch concentration and gelatinization. Carbohydr. Polym. 2007, 67, 73-79. [CrossRef]

16. Bilbao-Sainz, C.; Butler, M.; Weaver, T.; Bent, J. Wheat starch gelatinization under microwave irradiation and conduction heating. Carbohydr. Polym. 2007, 69, 224-232. [CrossRef]

17. Lewandowicz, G.; Fornal, J.; Walkowski, A. Effect of microwave radiation on physico-chemical properties and structure of potato and tapioca starches. Carbohydr. Polym. 1997, 34, 213-220. [CrossRef]

18. Mudgett, R. Dielectric properties of foods. Microw. Food Proc. Ind. 1985, 15-37.

19. Nelson, S.; Kraszewski, A. Grain moisture content determination by microwave measurements. Trans. ASAE 1990, 33, 1303-1307.

(C) 2015 by the authors; licensee MDPI, Basel, Switzerland. This article is an open access article distributed under the terms and conditions of the Creative Commons Attribution license (http://creativecommons.org/licenses/by/4.0/). 